\title{
Road Identification Through Efficient Edge Segmentation Based on Morphological Operations
}

\author{
Bandi Mary Sowbhagya Rani ${ }^{*}$, Vasumathi Devi Majety ${ }^{2}$, Chandra Shaker Pittala ${ }^{3}$, Vallabhuni Vijay ${ }^{4}$, \\ Kanumalli Satya Sandeep ${ }^{2}$, Siripuri Kiran ${ }^{5}$ \\ ${ }^{1}$ ECE, Vignan's Nirula Institute of Technology \& Science for Women, Guntur 522005, Andhra Pradesh, India \\ ${ }^{2}$ CSE, Vignan's Nirula Institute of Technology \& Science for Women, Guntur 522005, Andhra Pradesh, India \\ ${ }^{3}$ ECE, MLR Institute of Technology, Dundigal 500043, Hyderabad, Telangana, India \\ ${ }^{4}$ ECE, Institute of Aeronautical Engineering, Dundigal 500043, Hyderabad, Telangana, India \\ ${ }^{5}$ Department of CSE, Kakatiya Institute of Technology and Science, Warangal, Telangana 506015, India
}

Corresponding Author Email: ranibms@gmail.com

https://doi.org/10.18280/ts.380526

Received: 5 May 2020

Accepted: 12 September 2021

\section{Keywords:}

road identification, pixel intensity, image enhancement, segmentation, morphological operations, edge segmentation

\begin{abstract}
Road identification from high-precision images is important to programmed mapping, urban planning, and updating geographic information system (GIS) databases. Manual identification of roads is slow, costly, and prone to errors. Therefore, it is a hot topic among remote sensing experts to develop programmed techniques for road identification from satellite images. The main challenge lies in the variation of width and surface contents between roads. This paper presents a road identification and extraction strategy for satellite images. The strategy, denoted as ESMIRMO, recognizes roads in satellite images through edge segmentation, using morphological operations. Specifically, morphological operations were employed to enhance the quality of the original image, laying a good basis for accurate road detection. Next, edge segmentation was adopted to detect the road in the original image accurately. After that, the proposed strategy was compared with traditional methods. The comparison shows that our strategy could identify roads from satellite images more accurately than traditional methods, and overcome many of their limitations. Overall, our strategy manages to enhance the quality of satellite images, pinpoint roads in the enhanced images, and provide drivers and repairers with real-time information on road conditions.
\end{abstract}

\section{INTRODUCTION}

Road identification from satellite images is very important for traffic planning and disaster management in urban areas. Thanks to the advancement in high-precision satellite imaging, a huge volume of spatial information is now available [1]. How to extract and exploit the information precisely and proficiently is a hot topic in remote sensing [2]. Despite the high precision of satellite images, it is time-consuming and costly to identify roads amidst the extremely complex scenes. This calls for a programmed technique for road identification based on satellite images [3]. The programmed road identification technique would rely on various morphological operations to separate road segments, and extract the relevant pixels [4].

Roads are the backbone of urban traffic network. The identification of roads is essential to traffic control, urban management, road survey, and navigation [5]. It is a difficult yet important task to identify roads from satellite images. To facilitate road identification, the original images are often processed through simple spatial mapping, remote city identification, etc. Sometimes, segmentation techniques [6] are introduced to determine the region of interest, and distinguish between roads and the background. Figure 1 describes the general procedure of road identification.

Road systems are significant information sources of geographical information system (GIS) databases. The image data on roads should be extracted, and used to update GIS databases. The timely update helps with the query of traffic conditions, vehicle routing, urban planning [7], topographic mapping, as well as crisis management. For instance, the latest road data are needed to effectively handle traffic accidents or geographical disasters [8].
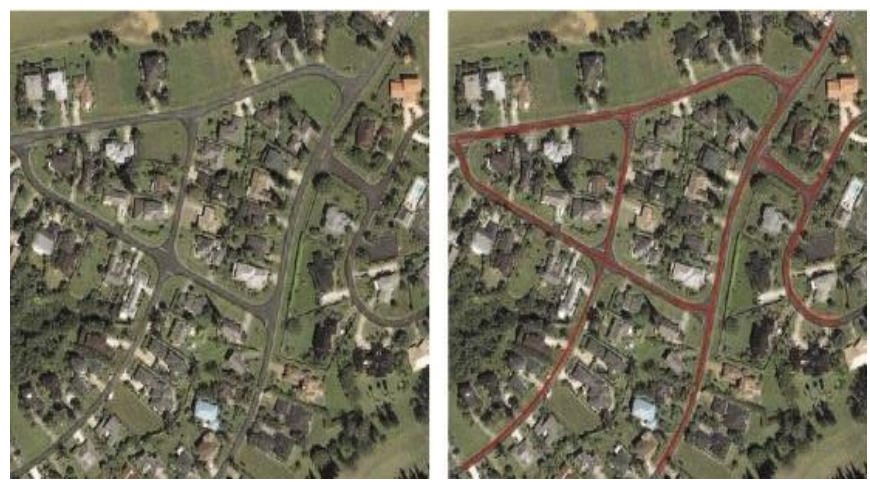

Figure 1. General procedure of road identification

The mapping of the road network relies on the accurate matching between boundary pixels of road segments. To improve the matching precision [9], the Canny operator is often adopted to remove the edges shorter than 50 pixels from the detected segments [10], and evaluate the remaining 
segments before matching. Specifically, the coordinates are calculated for each boundary pixel, as well as the maximum deviation of a segment between two junctions or endpoints [11]. If the maximum deviation is not acceptable, the corresponding edge is reduced repeated until the maximum deviation meets the requirement. In this way, the road boundaries are defined [12].

Numerically, the boundaries of an image object can be portrayed with straight lines or curves [13]. For example, roads, rivers, and coastlines are regularly represented by lines and curves in computerized mapping and GIS. Object representation in remote sensing is deemed as a hotspot in topographic mapping [14]. In many cases, small images called organizing components are employed to morphologically process the original image. The organizing component moves in a fixed way over the image to extract every pixel of the image object. Unsurprisingly, the organizing components do not apply to images containing directional pixels [15].

The road system in India mainly covers five levels of roads: expressways, national highways (NHs), state highways (SHs), district roads, and rural roads [16]. The expressways refer to the high-speed roads with four or more lanes. Ramps are needed for drivers to enter or exit the expressways. Most expressways in India are toll roads [17]. NHs refer to the roads between the biggest cities across the country. SHs link major cities within a state. District roads are those linking up the villages in a taluka. Rural roads are the unestablished roads in villages [18].

With the help of Google Maps and other online databases, the user can find the shortest route from place A to place B. However, the online databases are not updated in real time. For example, if a road between places A and B is being repaired or under construction, Google Maps might still recommend the user to go through that road [19], relying on the outdated satellite images. To avoid the problem, the user could check road maps, which are manually generated and modified based on high-resolution aerial images. But these maps are too expensive and time-consuming to maintain [20].

Morphological operations provide an advantageous and effective strategy to improve image quality. The specific operation must be selected according to the actual conditions. In urban areas, roads and structures are highlights on satellite images [21]. By remote sensing, the road condition could be captured with a high cost-effectiveness, which has a major impact on the daily activities of the urban population [22].

To timely refresh the information of the road network, this paper proposes a strategy to extract roads from an enormous scope of satellite images on a city [23]. Our strategy characterizes and recognizes each object in the original image by five kinds of attributes: photometric, geometric, topological, practical, and relevant attributes. These attributes are widely used by manual road identification approaches. The effectiveness of our strategy was demonstrated through tests, using pervasive local and practical road properties like material and surface profile.

The following assumptions were put forward before implementing our strategy: the road surface is typically homogenous, and different from the nearby objects; a road is extended, has a greatest shape, and does not end without a reason; the roads cross each other, forming a network, and link up different places; a road can be identified by a unique distribution of trees.

\section{LITERATURE REVIEW}

Road identification models usually contain two submodules: the first module is responsible for detecting straight segments; the second one is responsible for defining road contours.

Programmed road identification focuses on the positioning of key places. Firstly, the original image is preprocessed to remove the noises, and enhance the contrast, making it easier to partition the roads from the background. The distribution of road regions is assumed to be recognizable by image histogram [24]. The rough road cover could be detected through Otsu's thresholding on the grayscale image. On this basis, the edges could be extracted through morphological reconstruction and boundary tracking.

Progressive explicit road identification emphasizes the center of each satellite image, and assumes that roads have homogenous properties. Under this assumption, it is possible to differentiate road surfaces from road lines. For this reason, the original image is generally segmented through the homogram segmentation system, using both spatial attribute and image quality. Before creating homograms, Gaussian smoothing is performed on the original image to remove outliers and determine the most suitable shape of homograms. After homogram segmentation, morphological operations are needed to improve the accuracy of the detected road contours. To identify roads in satellite images, it is strongly recommended to integrate scientific morphological operations with dynamic shape model, and use Bayesian network to track the road states (route, and direction) after the road profile has been recognized manually.

The perception model for road identification coordinates the existing road profile with the reference model. It outperforms many programmed road identification methods, as indicated by the test results on images captured by the enhanced thematic mapper (ETM) of Landsat (resolution: 15m). Treating roads as long and narrow structures with significant pixel differences, the perception model can quickly characterize the fundamental attributes of roads, including geometric, radiometric, topologic, and other relevant attributes The road edges in the original images can be tracked by the double edge following strategy. Then, the fake positives can be disposed of based on the homogeneity and basic properties of roads. Experimental results show that the perception model applies to various types of satellite images.

Considering the directions, width limits, and varied properties of roads, the artificial calculation segment division method firstly differentiates images with a lateral operator called the beam circle operator, and then removes false positively with a Bayesian model. In this way, each original image can be divided into road areas and non-road areas. After that, the common technique of toe estimation is called to remove the highlights, and distinguishes the direction between road and non-road areas. In the Bayesian model, a lognormal operation is developed based on the region-to-perimeter proportions of road reproductions.

The versatile pixel-based strategy distinguishes the edges of the original image, and uses the data in boundary calculation, revealing the regions of interests. On this basis, the starting points are chosen with the aid of setting features, including but not limited to contiguousness, parallelism, oppositeness, and crossing point of line fragments. 
Yassemi et al. [1] proposed the backpropagation (BP) calculation, marking a great step forward in road identification based on neural networks. For the most part, the BP calculation relies on the BP neural network to directly characterize the unearthly and contextual data from the original images. To delineate road centerlines, Yadav and Agrawal [3] presented a powerful method by preparing a neural system based on spectral and edge data. The system could come up with great outcomes, although the extraction results contain many non-road edges.

Support vector machine (SVM) is a supervised learning strategy for feature characterization. By this strategy, the linear road can be recognized in a remote sensing image through two steps. To start with, the road segments can be identified with a direct locator. Then, the genuine road areas could be chosen and connected, according to the extracted features. Kahraman et al. [5] combined Marakov random surface model with the SVM classifier into an organized road extractor. To deal with the spectral homogeneity, the selfloader road identification technique utilizes texture features, which need to be extracted manually. This defect can be solved with the help of SVM. Many cases have shown that SVM is more precise, and powerful in object detection than radial basis function (RBF) and k-nearest neighbors (k-NN) algorithm.

In the past three decades, many scholars have explored programmed road identification. For example, Liu et al. [6] derived a technique that includes powerful programming. Miao et al. [9] recommended a road identification strategy, which improves the original image by level set technique, selects a starting point from the road, improves the extracted segments through iterative smoothing, and pinpoints the road centerlines. Ma et al. [11] developed a road area recognition model with two modules. The first module reduces the image by computing the mean and difference of Gaussian distribution, while the second module separates the road area from the original image, enhances the road recognition, and applies fuzzy strategy.

\section{METHODOLOGY}

Road identification from a given image generally consists of several stages: enhancement, denoising, segmentation, morphological operations, feature extraction, and testing the segmentation effect.

Before applying an edge detector, it is necessary to eliminate the noises by smoothing the original image with a low-pass filter, because the edges are the main difficulty in image denoising. In this paper, a $3 \times 3$ operator is adopted to separate the pixels, reduce the noises, and enhance the saliency of distant pixels, making the original image smoother.

\subsection{Image enhancement}

To improve road identification, different types of road images are subjected to contrast enhancement. Firstly, median filtering is performed to remove the noises. The median filter $\mu$ can be expressed as:

$$
\mu_{m e d}^{2}=\frac{1}{2 p t^{2}(p)} \approx \frac{\pi_{i}^{2}}{n+\frac{\pi}{2}} \cdot \frac{\pi+1}{2}+\mathrm{k}
$$

where, pt is an image pixel; $\pi$ is the contrast increment of a pixel through enhancement; $\mathrm{k}$ is a constant; $\mathrm{n}$ is the total number of pixels in the original image.

$$
\begin{gathered}
p_{i}^{\prime}=\sum i_{2 i+k} a t_{k+} \mathrm{b} t_{k} \\
p_{a t / 2+i}^{\prime}=\sum a_{2 i+k} * \mu \\
p_{b t / 2-1}^{\prime}=\sum i_{2 i+k} a t_{k+} \mathrm{b} t_{k}+\sum a_{2 i+k} * \mu
\end{gathered}
$$

The pixels from the image can be extracted as:

$a$ is a pixel extracted from the image; $b$ is a neighbor pixel of $a$; $I$ is the original image; $t$ is the threshold; $\mu$ is the depth of the pixel; $\mathrm{k}$ is the constant.

$$
P_{i, j}=\exp \left(\frac{-\left(T_{n}(a)-\mathrm{T}_{r}(b)\right)^{2}}{\left(\frac{2 \beta^{2}}{2}\right)}\right)+\sum I_{i+a / 2+b}^{\prime} v_{n}
$$

The dark pixels are extracted from each image. Only the low-contrast pixels are enhanced to improve road identification. The pixel quality can be improved by:

$\mathrm{T}$ is the contrast threshold; $\mathrm{Pi}$ is the intensity of pixels in image $\mathrm{i} ; \beta^{2}$ is the increment of intensity; $\mathrm{V}$ is the pixel vector of image i.

\subsection{Edge segmentation}

The right segmentation technique is critical to the division between road pixels and non-road pixels in the original image. Our strategy allocates relevant edge pixels into the same cluster, and divides the original image into sub-images by extracting relevant edge features from the image:

$$
I(a, b)=\sum_{i=1}^{n} \sum_{j=1}^{n} S_{i k}^{r} p^{r}\left(a_{i}, n_{i}\right)
$$

where, $S_{i k}$ is the k-th p-dimensional data vector; $n_{i}$ is cluster center $\mathrm{i}, p_{r}$ is the membership of $a n_{i}$ to the $\mathrm{i}$-th cluster; $\mathrm{n}$ is the weight vector of each edge pixel. The dilation operation $\oplus$ is performed on the extracted pixels:

$$
\mathrm{a} \oplus \mathrm{b}=\{\mathrm{N} \mid \mathrm{bi} \cap \mathrm{a} !=\phi\}
$$

After dilation, image segmentation is performed on relevant and irrelevant edge pixels:

$$
\begin{aligned}
I(a, b)=\sum_{k=1}^{n} \frac{\left(a_{k j}, w_{i j}\right)}{2} & \\
& +\sum_{j=1}^{n} S_{i k}^{r} b^{r}\left(a_{i}, n_{i}\right) \oplus S_{i k}^{r}
\end{aligned}
$$

where, $w_{i j}$ is the weight vector of cluster centers; $\mathrm{S}$ is the cluster for pixels $i$ to $\mathrm{n}$ based on the weights; $\mathrm{a}$ and $\mathrm{b}$ are a pixel and its neighoring pixel, respectively.

Then, the erosion operation $\Theta$ is performed on the pixels: 


$$
\mathrm{a} \ominus \mathrm{b}=\left\{\mathrm{N} \mid \mathrm{bi} \cap \mathrm{a}^{\mathrm{i}}=\phi\right\}
$$

Let $I(x, y)=\left\{x_{1}, x_{2} \in N i\right\}$ be the set of estimation vectors for the neighbors of pixel $\mathrm{i}$, where $\mathrm{Ni}$ is a neighbor of pixel I. The density and darkness of edge pixels are characterized to the entirety of multivariate Gaussian distribution with intensity $\mathrm{Yi}^{\prime}$ and covariance $\Sigma \mathrm{i}^{\prime}$.

$$
\begin{aligned}
P\left(Y_{i}\right)=\frac{1}{2 \pi^{-\mathrm{D} / 2}} \exp \left\{-\frac{1}{2}\left(Y_{i}\right.\right. \\
\left.\left.\quad-Y_{i}\right)^{T} \sum_{i}^{-1}\left(Y_{i}-Y_{i}\right)+I(a, b)\right\} \\
\overline{P_{i}}=\frac{1}{\pi N_{i}} \sum_{i \in N_{i}} \beta Y_{i} * \sum_{i+a / 2+b}^{\prime} v_{n} \ominus \sum I_{i+a / 2+b}^{\prime}
\end{aligned}
$$

where, $\pi N_{i}$ is the number of elements within set $N_{i}$. Based on the original image, the road intensity can be calculated as $\mathrm{Y}=\left\{\mathrm{Y}=\mathrm{p}\left(X_{i}\right): \mathrm{i}=1, \ldots, \mathrm{N}\right\} . Y_{i}$ is normalized to $[0,255]$, and rounded to the nearest integer.

After the original image is segmented, a few classes are acquired which speak to urban and nonurban objects. Then, the objects of road identification are selected, and subjected to a dull and equivalent number of morphological operators. The most important operators are responsible for disintegration and extension. The features for road identification can be expressed as:

The peak signal to noise ratio (PSNR) can be calculated to identify the mean squared error (MSE):

$$
\mu_{n}=\max \left(\frac{\sigma\left(N T_{n}^{l}\right)}{\sigma\left(I^{l}\right)}, \frac{\sigma\left(I^{l}\right)}{\sigma\left(N T_{n}^{l}\right)}\right)+\sum_{j=1}^{n} S_{i k}^{r} p^{r}\left(a_{i}, n_{i}\right)
$$

The Peak Signal to Noise Ratio is calculated for identifying Mean Square Error (MSE) that is calculated as:

$$
M S E=\frac{\sum_{a=1}^{x} \mathrm{p} \sum_{b=1}^{y} \mathrm{q}(\mathrm{T}(a, b)-G(a, b))^{2}}{x * y}
$$

\section{RESULTS}

The proposed strategy was compared with traditional methods on images from URL https://www.cs.toronto.edu/ vmnih/data/mass_roads/train/sat /index.html. The comparison shows that our strategy could detect road accurately from the images in a shorter time than the traditional approaches. Table 1 lists the parameters for road identification.

The road images from the URL were enhanced to improve the quality of road identification. The process of image enhancement is depicted in Figure 2.

Table 2 compares the accuracy of road identification between our method and existing models.

Despite the image processing, there were still some openings and noises in the roads, because it is almost impossible to differentiate the relevant segments from small ground artifacts, such as buildings and vehicles. To further improve the precision of road identification, the images were further treated through morphological operations like opening, closing, extension, and disintegration. Through these

operations, salient objects, i.e., the roads could be recognized by their type. Figure 3 shows how to extract the features for road recognition. Figure 4 illustrates the road identification from the images.

The road detection time from the image in the proposed method is compared with traditional manual digitization method and the time levels are depicted in Figure 5.

The Accuracy levels of the proposed method and traditional methods are depicted in Figure 6. The results show that the proposed method accurately identifies the roads from the input image dataset.

Table 1. Parameters for road identification

\begin{tabular}{ccccc}
\hline Completeness & Correctness & Sensitivity & Specificity & Accuracy \\
\hline 94.26 & 97.85 & 94.75 & 93.28 & 92.38 \\
\hline
\end{tabular}
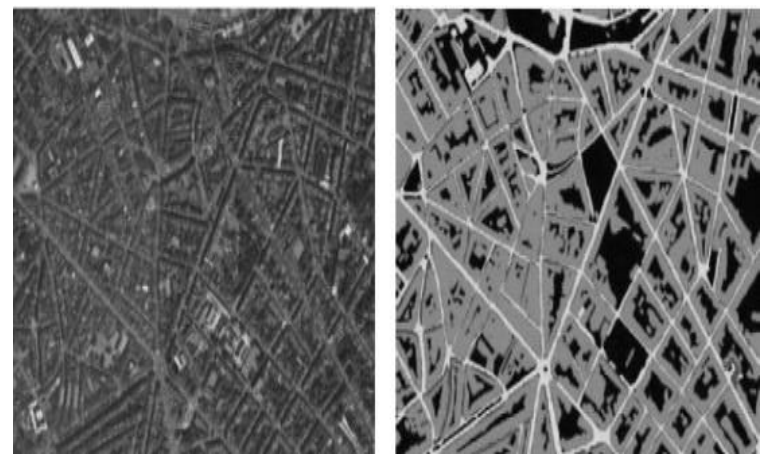

Figure 2. Image enhancement

Table 2. Comparison of accuracy

\begin{tabular}{ccc}
\hline Model & Features & Accuracy \\
\hline SVM & Intensity, length & 63.5 \\
ANN & Intensity, edge, spectrum & 73 \\
Mean shift & Histogram of the image & 81 \\
Proposed & Intensity, edge gradient, length, & \multirow{2}{*}{ width, Mean intensity value, } \\
ESMIRMO & texture & 96 \\
\hline
\end{tabular}
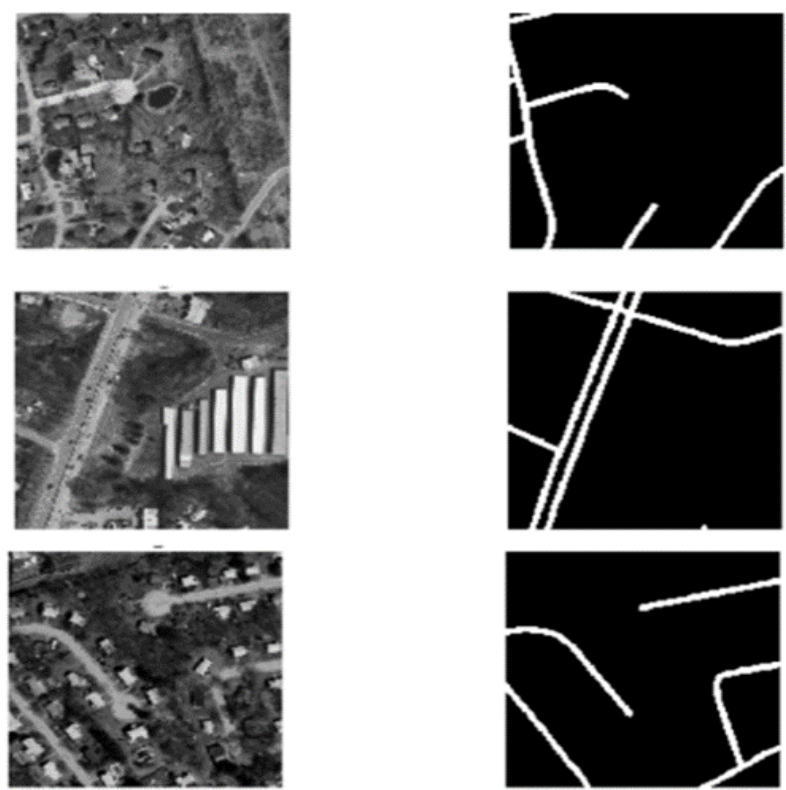

Figure 3. Road feature extraction 


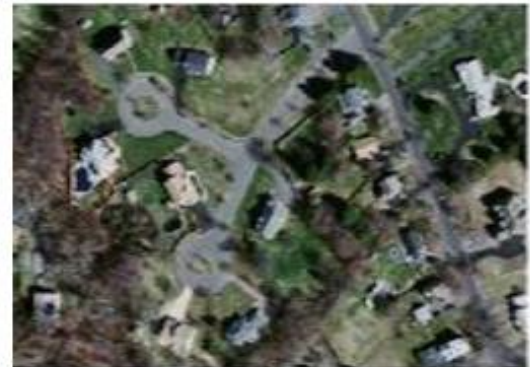

Initial Image

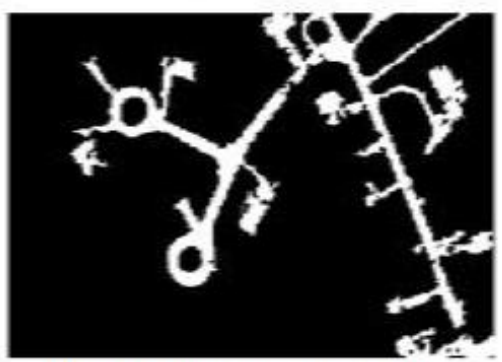

After filtering by size of segments

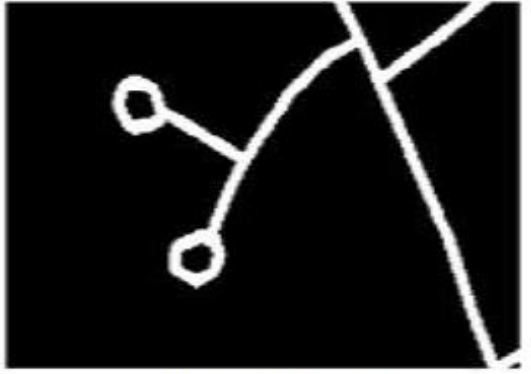

Ground Image

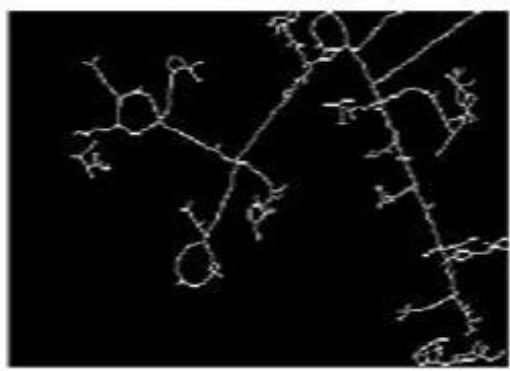

Skeleton

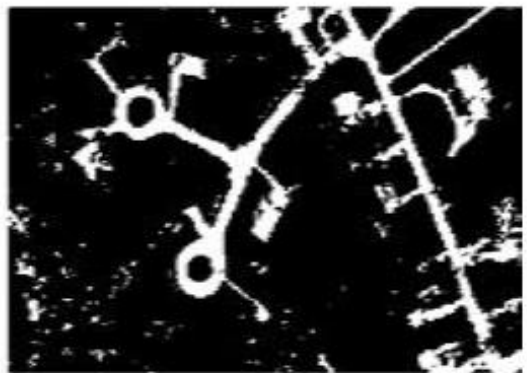

Edge Segmentation Result

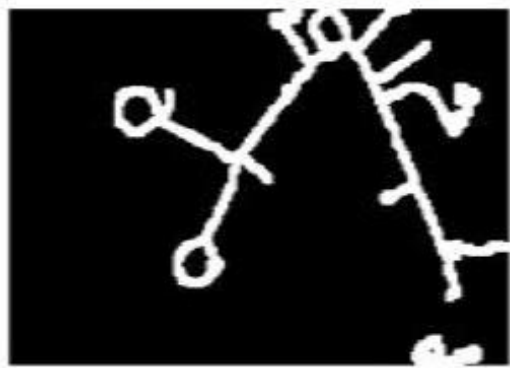

Road Network

Figure 4. Road identification process from image

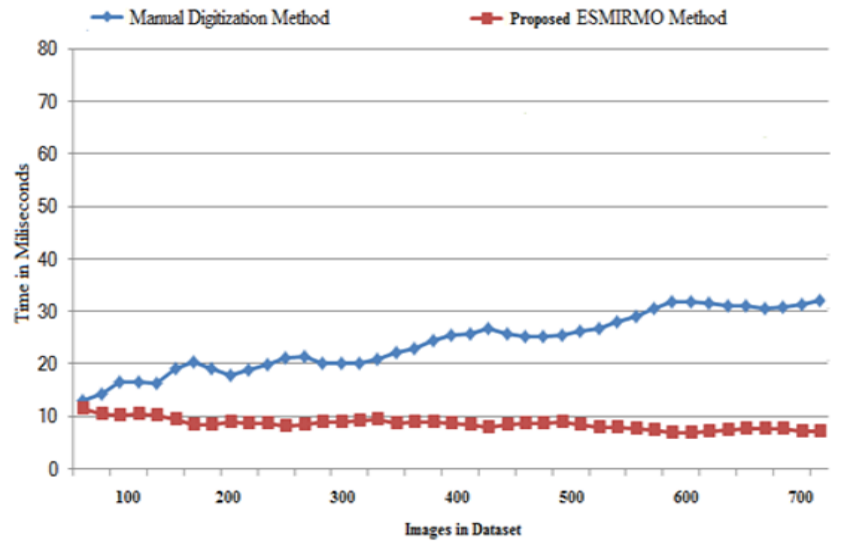

Figure 5. Time levels in road identification

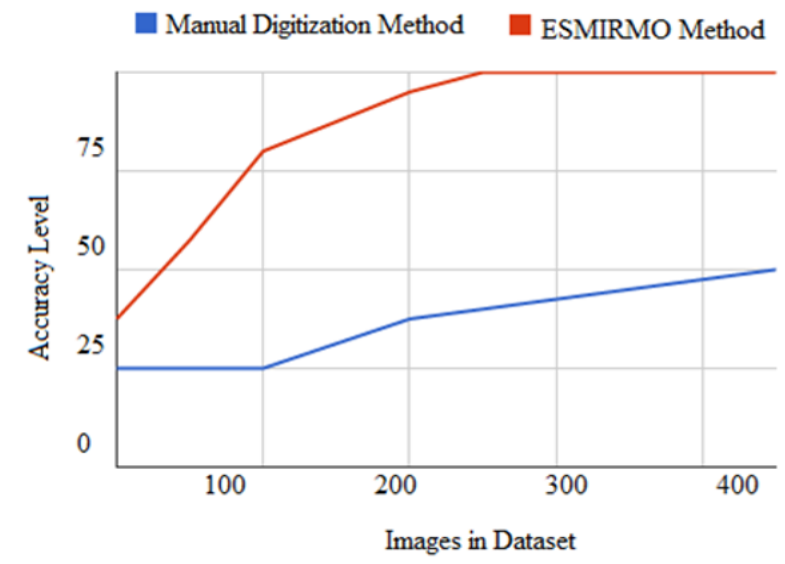

Figure 6. Accuracy level

\section{CONCLUSIONS}

If the color information is available, it can provide valuable information to detect objects. We gain from color knowledge in terms of chromaticity values in the proposed process. Color is not mentioned anywhere in the main text. Probably, we could remove this part to the "directions of future research" at the end of Conclusions.

Remote sensing is a cost-effective approach to understand the situation of bridges, roads, and other land transport facilities, and learn about the actual transport conditions. Because of complexity and resolution problems, the earlier methods cannot successfully detect roads in complex aerial images. To solve the problem, this paper proposes an automatic and rapid method for road detection in satellite images, and demonstrates the usefulness of our method in practice. In our strategy, edge segmentation is cleverly integrated with morphological operations to improve the original images, and enable accurate identification of roads. Experimental results show that our strategy can achieve $92 \%$ precision in road identification. In future, more features, e.g., color and fewer edge detection attributes will be considered to further improve the precision of our strategy.

\section{REFERENCES}

[1] Yassemi, S., Dragićević, S., Schmidt, M. (2008). Design and implementation of an integrated GIS-based cellular automata model to characterize forest fire behaviour. Ecological Modelling, 210(1-2): 71-84. https://doi.org/10.1016/j.ecolmodel.2007.07.020

[2] Wang, W., Yang, N., Zhang, Y., Wang, F., Cao, T., Eklund, P. (2016). A review of road extraction from remote sensing images. Journal of Traffic and Transportation Engineering (English Edition), 3(3): 271282. https://doi.org/10.1016/j.jtte.2016.05.005

[3] Yadav, P., Agrawal, S. (2018). Road network identification and extraction in satellite imagery using Otsu's method and connected component analysis. International Archives of the Photogrammetry, Remote Sensing \& Spatial Information Sciences. https://doi.org/10.5194/isprs-archives-XLII-5-91-2018

[4] Ma, R., Wang, W., Liu, S. (2012). Extracting roads based 
on Retinex and improved Canny operator with shape criteria in vague and unevenly illuminated aerial images. Journal of Applied Remote Sensing, 6(1): 063610. https://doi.org/10.1117/1.JRS.6.063610

[5] Kahraman, I., Turan, M.K., Karas, I.R. (2015). Road detection from high satellite images using neural networks. International Journal of Modeling and Optimization, 5(4), 304.

[6] Liu, X., Wang, X., Wright, G., Cheng, J.C., Li, X., Liu, R. (2017). A state-of-the-art review on the integration of Building Information Modeling (BIM) and Geographic Information System (GIS). ISPRS International Journal of Geo-Information, 6(2): 53. https://doi.org/10.3390/ijgi6020053

[7] Deepan, P., Abinaya, S., Haritha, G., Iswarya, V. (2018). Road recognition from remote sensing imagery using machine learning. International Research Journal of Engineering and Technology, 5(3): 3677-3683.

[8] Liu, J., Qin, Q., Li, J., Li, Y. (2017). Rural road extraction from high-resolution remote sensing images based on geometric feature inference. ISPRS International Journal of Geo-Information, 6(10): 314. https://doi.org/10.3390/ijgi6100314

[9] Miao, Z., Wang, B., Shi, W., Zhang, H. (2014). A semiautomatic method for road centerline extraction from VHR images. IEEE Geoscience and Remote Sensing Letters, 11(11): 1856-1860. https://doi.org/10.1109/LGRS.2014.2312000

[10] Duan, X., Zhang, Y., Guo, Z. (2017). Feature extension of cluster analysis based on microblog. Computer Engineering and Applications, 53(13): 90-95.

[11] Ma, H.F., Zeng, X.T., Li, X.H., Zhu, Z. (2016). Short text feature extension method of improved frequent term set. Computer Engineering, 42: 213-218. https://doi.org/10.3969/j.issn.1000-3428.2016.10.037

[12] Wang, R.J., Wang, K., Zhang, F., Gao, J.J., Li, Y., Yue, T. (2017). Emission characteristics of vehicles from national roads and provincial roads in China. Huanjing Kexue, $\quad 38(9)$ : 3553-3560 https://doi.org/10.13227/j.hjkx.201701087

[13] Ren, S., He, K., Girshick, R., Sun, J. (2016). Faster RCNN: Towards real-time object detection with region proposal networks. IEEE Transactions on Pattern Analysis and Machine Intelligence, 39(6): 1137-1149. https://doi.org/10.1109/TPAMI.2016.2577031

[14] Gao, L., Shi, W., Miao, Z., Lv, Z. (2018). Method based on edge constraint and fast marching for road centerline extraction from very high-resolution remote sensing images. Remote Sensing, 10(6): 900. https://doi.org/10.3390/rs10060900

[15] Liu, L., Lim, S. (2017). Color component-based road feature extraction from airborne lidar and imaging data sets. Journal of Surveying Engineering, 143(1): 04016021. https://doi.org/10.1061/(ASCE)SU.19435428.0000198

[16] Khawaldeh, S., Pervaiz, U., Rafiq, A., Alkhawaldeh, R.S. (2018). Noninvasive grading of glioma tumor using magnetic resonance imaging with convolutional neural networks. Applied Sciences, 8(1): 27. https://doi.org/10.3390/app8010027

[17] Zhao, Z.Q., Bian, H., Hu, D., Cheng, W., Glotin, H. (2017). Pedestrian detection based on fast R-CNN and batch normalization. In International Conference on Intelligent Computing, pp. 735-746. https://doi.org/10.1007/978-3-319-63309-1_65

[18] Ma, Y., Chen, H., Liu, G. (2018). General mean pooling strategy for color image quality assessment. Laser Optoelectron. Prog. 55(2): 021007.

[19] Shao, X.X., He, X.Y. (2017). Statistical error analysis of the inverse compositional Gauss-Newton algorithm in digital image correlation. In International Digital Imaging Correlation Society, pp. 75-76. https://doi.org/10.1007/978-3-319-51439-0_18

[20] Birtea, P., Comănescu, D. (2017). Newton algorithm on constraint manifolds and the 5-electron Thomson problem. Journal of Optimization Theory and Applications, 173(2): 563-583. https://doi.org/10.1007/s10957-016-1049-0

[21] Narayana, V.L., GOPIL, A.P. (2017). Visual cryptography for gray scale images with enhanced security mechanisms. Traitement du Signal, 34(3-4): 197-208. https://doi.org/10.3166/ts.34.197-208

[22] Gopil, A., Narayana, V.L. (2017). Protected strength approach for image steganography. Traitement du Signal, 35(3-4): 175-181. https://doi.org/10.3166/TS.34.175181

[23] Yin, C., Dadras, S., Huang, X., Mei, J., Malek, H., Cheng, Y. (2017). Energy-saving control strategy for lighting system based on multivariate extremum seeking with Newton algorithm. Energy Conversion and Management, 142: 504-522. https://doi.org/10.1016/j.enconman.2017.03.072

[24] Lyashenko, V.V., Babker, A.M.A.A., Kobylin, O.A. (2016). The methodology of wavelet analysis as a tool for cytology preparations image processing. Cukurova Medical Journal, 41(3): 453-463. https://doi.org/10.17826/cukmedj.237468 